\title{
Sixteen months' experience with 140 posterior chamber intraocular lens implants
}

\author{
JOHN L. PEARCE
}

SUMMARY Three main designs of posterior chamber lenses in 140 cases have been reviewed. The visual results are satisfactory; patient satisfaction is good; and postoperative complications are limited to anterior dislocation of the feet of the lens and the necessity to wash out cortical remnants from behind the prosthesis in a small percentage of cases. The posterior chamber prosthesis is free from some of the complications of other intraocular lenses and probably provides the most 'normal' looking eye of any pseudophakoi.

The author has reported (Pearce, 1976) a small series of patients in whose eyes lightweight posterior chamber intraocular lenses were implanted. This paper considers further experience in 140 cases with modifications in lens design and in surgical technique.

In America cataract surgery by the extracapsular technique has recently been favoured, particularly since the advent of phaco-emulsifying and aspiration irrigation techniques, which facilitate the complete removal of cortical remnants. The equipment, however, is expensive and is likely to be available in only a limited number of European centres.

The pathogenesis of aphakic cystoid macular oedema is still speculative, although two types have been recognised. The Irvine-Gass syndrome of transient macular oedema occurs in up to $40 \%$ of cataract extractions, and the Jaffe syndrome of definite cystoid macular changes without oedema, wherein recovery of visual function is often limited, occurs less frequently. The incidence of cystoid macular oedema in pseudophakia is variously reported as being between 5 and $12 \%$, with a higher incidence after intracapsular cataract extraction. Binkhorst et al. (1976) stated that their series showed a definite superiority of extra- over intracapsular cataract surgery so far as the long-term protection of the retina was concerned, with special reference to cystoid maculopathy and retinal detachment. Capsular fixation, as contrasted with iris support lenses, has an increasing number of advocates, though capsular fixation is not always successfully attainable. Inert iris suture materials such as poly-

Address for reprints: Mr J. L. Pearce, Windrush House, 4 Hayshire Lane, near Kidderminster, Worcestershire propylene and platinum-iridium wire avoid the disintegration of iris sutures with time.

It seems likely that in future a balanced salt solution incorporating gluthathione and adenosine will become available for anterior chamber irrigation, because it reduces postoperative corneal oedema and the risk of endothelial damage.

\section{Current design of Pearce bipod and tripod posterior chamber lens}

Different lengths of the fenestrated haptic feet are available, otherwise the basic design of the lens (Fig. 1, Table 1) is unchanged, being fabricated of polymethylmethacrylate (PMMA) with a 4-mm optic of lens power equivalent to a spectacle lens of $+11.50 \mathrm{D}$ at a vertex distance of $10 \mathrm{~mm}$ or a thin lens of 21.0 D in aqueous. Shortly, lenses of 19 and 23 dioptre in aqueous will be available; the weight of the lens remains between 8 and $9 \mathrm{mg}$ in air.

The bipod lens is generally used for secondary insertion through a small incision after prior extracapsular extraction. It can also be used combined with penetrating keratoplasty, being sutured by a double Pearce iris stitch.

The tripod lens is normally inserted in an inverted ' $Y$ ' fashion with the superior limb directly sutured through the fenestration to the iris. Choice of length of inferior limbs depends on the pupil size after extracapsular extraction is completed. If the pupil remains large, it is wise to insert a lens with $5 \mathrm{~mm}$ radial inferior feet, otherwise anterior dislocation of the inferior limbs may occur postoperatively. A tripod lens is now available with $4.75 \mathrm{~mm}$ radial limbs, which can be used without iris fixation 


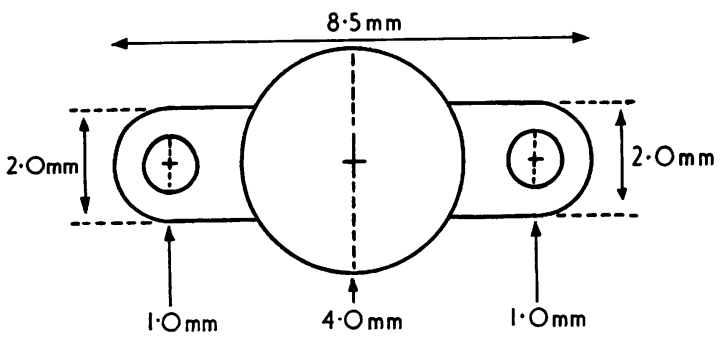

으

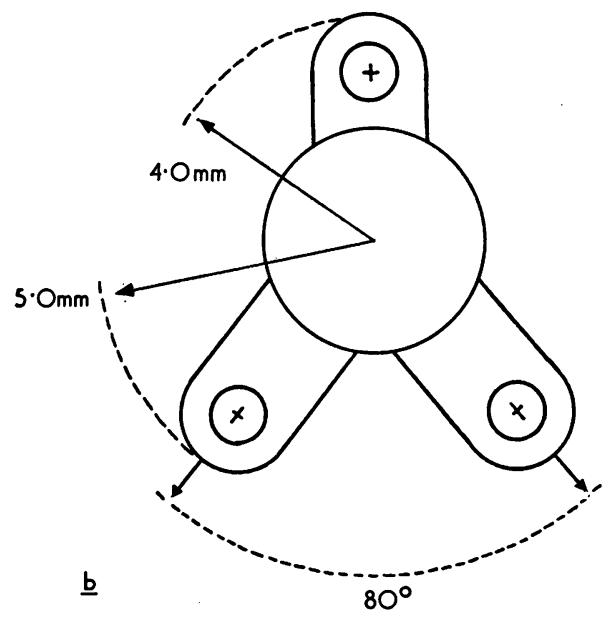

Table 1 Design of lenses

\begin{tabular}{lllll}
\hline Type & Form & $\begin{array}{l}\text { Angular separation } \\
\text { Inferior limbs }\end{array}$ & $\begin{array}{l}\text { Superior } \\
\text { limbs }\end{array}$ & $\begin{array}{l}\text { Inferior } \\
\text { limbs }\end{array}$ \\
\hline 493A & Bipod & - & $\begin{array}{l}(\mathrm{mm}) \\
4.25\end{array}$ & $\begin{array}{l}(\mathrm{mm}) \\
4.25\end{array}$ \\
493B & Tripod & $80^{\circ}$ & 4.00 & 4.50 \\
493C & Tripod & $80^{\circ}$ & 4.00 & 5.00 \\
493D & Tripod & $120^{\circ} \mathrm{C}$ & 4.75 & 4.75 \\
493E & Tripod & $80^{\circ}$ & 4.75 & 4.75 \\
\hline
\end{tabular}

All limbs $0.25 \mathrm{~mm}$ thick, $2.0 \mathrm{~mm}$ wide; fenestration $1.0 \mathrm{~mm}$.

Standard optic diameter $4.00 \mathrm{~mm}$ equiconvex.

Standard powers: In aqueous +21.50D equivalent spectacle correction $+\mathbf{1 2 . 0 0}$.

Other powers: In aqueous +19.00D equivalent spectacle correction +11.00 ; in aqueous +23.00D equivalent spectacle correction $+13 \cdot 00$.

provided adequate anterior capsule is left below to allow capsular fixation.

\section{Surgical technique}

All surgery is performed under the operating microscope with continuous irrigation of the cornea with Ringer's solution.

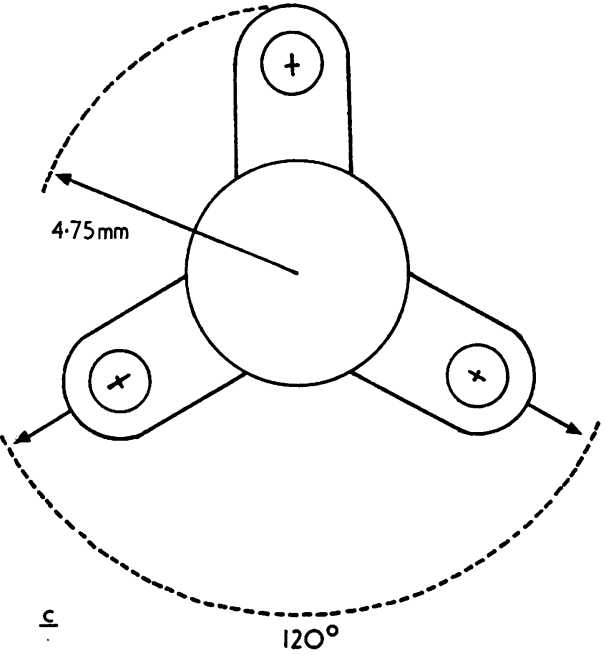

Fig. 1

PREOPERATIVE TREATMENT

Maximum mydriasis is obtained by topical instillation of cyclopentolate $1 \%$ with phenylephrine $10 \%$.

\section{OPERATION}

(a) A scissor incision is made of a small limbalbased conjunctival flap.

(b) A 9-mm, two-thirds thickness perpendicular (100 degrees) groove is incised with a diamond knife $1 \mathrm{~mm}$ from the surgical limbus.

(c) Three 10/0 Perlon corneal stitches are preplaced in the gutter and looped out of the wound.

(d) The anterior chamber is entered with a small incision by the diamond knife.

(e) A hooked-over 22-gauge hypodermic needle attached to a 5-ml syringe filled with Ringer's solution is used as a cystitome. Multiple closely placed rotary tears around the anterior capsule about $1 \mathrm{~mm}$ from the equator of the lens are performed. This is the 'beer can' technique of anterior capsulotomy.

(f) The eye is then opened with the diamond knife and the anterior capsule removed with Arruga's intracapsular forceps. 
(g) The nucleus is dislocated into the anterior chamber using a lachrymal cannula attached to a syringe filled with Ringer's solution. The tip of the cannula can be passed partly underneath the nucleus, so that the nucleus is partly washed into the anterior chamber.

(h) A small vectis is passed underneath the nucleus and the nucleus lifted out of the anterior chamber. The remaining cortical fragments are washed out, it being wise to avoid aspiration unless the depth of the anterior chamber is maintained. Even with a slit-lamp beam it may be difficult to distinguish between a thin clear layer of posterior subcapsular cortical material and the posterior capsule itself. With experience this layer can be peeled off, using an irrigating cannula or a cannula with a sand-blasted tip.

(i) Pearce iris stitch. The iris sphincter is gripped with the suture-tying forceps at 12 o'clock. 5-mm corneoscleral needle swaged to polyamide, polypropylene, or platinum/iridium wire is passed through the pupil behind the iris. The point of the needle emerges at 12 o'clock some $2 \mathrm{~mm}$ from the limbus (when about half the chord length of the needle is covered by iris) and is then laid aside. The loose ends of the iris stitch are placed through the fenestration of the haptic foot of the lens and tied, the free ends being excised.

(j) The anterior chamber is filled with air and the lens is placed within the capsular bag using the special introducing forceps (air within the anterior chamber facilitates the identification of the anterior capsule remnants and also acts as a buffer between the prosthesis and the corneal endothelium).

(k) The iris at 12 o'clock is placed over the superior limb of the prosthesis, and another bite of the iris is taken circumferentially just behind or in front of where the stitch emerges from behind the iris, leaving a small loop; this is tied and the ends are cut with curved Vannas scissors.

(l) Provided adequate anterior capsulectomy and removal of cortex has been performed there seems to be little need for a peripheral iridectomy. Corneoscleral sutures are tied, air is removed from the anterior chamber, and this is refilled with Ringer's solution, the lens being centred in the optical axis with an air cannula. The conjunctival flap is closed.

(m) Subconjunctival depot steroid with an antibiotic is injected into the lower fornix; eyelids are closed after removal of the plastic drape, and a pad and cartella are placed over the eye.

\section{POSTOPERATIVE TREATMENT}

The first dressing is performed the following morning, the patient being allowed up as soon as the effect of the anaesthetic has worn off. Topical steroids and short-acting mydriatic/cycloplegic drops such as cyclopentolate are instilled for 2 to 3 weeks to keep the pupil active. The return of visual function is rapid provided there is no lens matter behind the implant.

\section{Operative complications}

Vitreous loss. Expression of the nucleus was followed by vitreous loss in 2 of the earlier cases with Binkhorst posterior chamber lenses.

Case 1. Present visual acuity is $6 / 6$ unaided. The patient developing cystoid maculopathy which resolved after a course of indomethacin.

Case 2. Present visual acuity $6 / 1815$ months after surgery. This case has a dry senile macular degeneration.

There have been no other serious operative complications. However, removal of all cortical remnants, especially in an immature cataract, can be time-consuming, it being important to use a microscope with coaxial illumination perpendicular to the cornea.

EARLY POSTOPERATIVE COMPLICATIONS

Implant dislocation. Anterior dislocation of 1 or 2 of the inferior feet of the implant has been noted in 4 eyes $(3 \%)$ within 2 days of surgery. This may have been due to immediate excessive postoperative pupillary dilatation, and it has not been possible to reposition any of these lenses medically. All have been repositioned surgically under local anaesthetic through a small stab incision in the cornea without retrobulbar anaesthesia. Posterior dislocation of the lens has been absent in this series.

Striate keratitis has become increasingly less noticeable with improvement in extracapsular technique, avoidance of aspiration of cortical remnants, and reduction of the size of the corneoscleral wound from 170 to 100 degrees.

Surgical uveitis has been minimal, with no case of hypopyon or hyphaema. The absence of a peripheral iridectomy possibly contributed to the lack of haemorrhage.

LATE POSTOPERATIVE COMPLICATIONS

Bullous keratopathy. One case has bullous keratopathy of the upper third of the cornea. The patient wears a soft contact lens and has a corrected vision of $6 / 96$ months after surgery. In addition there is one further case of corneal oedema in an eye with 
traumatic cataract, but the oedema is gradually subsiding.

Decentred lenses. Nine eyes $(6 \%)$ were found to have the lens too far from the visual axis and required centration by means of a McCannel iris stitch.

\section{McCannel iris stitch technique}

(a) Local anaesthesia is given, with facial nerve akinesia.

(b) A diamond knife is used to cut a $2-\mathrm{mm}$ corneal incision $2 \mathrm{~mm}$ from the limbus adjacent to the theoretical position of the implant.

(c) A 5-mm iris needle swaged to polypropylene, polyamide, or platinum/iridium wire is passed through the incision, and through and behind the iris and inferior foot of the lens, the point of the needle passing through the fenestration in the lens foot, which can be seen silhouetted against the iris, then out through the iris and cornea by counterpuncturing.

(d) A hooked hypodermic needle attached to a syringe filled with Ringer's solution is passed through the original incision. The suture within the anterior chamber is picked up by the needle hook and pulled back out of the eye.

(e) The suture is tied, the ends cut flush with the knot, and the anterior chamber reformed with balanced salt solution.

Capsulotomies. Condensation and fibrosis of the posterior capsule have been rare in this short followup. However, 12 eyes $(8.5 \%)$ have required or will require irrigation of cortical remnants which were behind the prosthesis. Generally this can be performed under local anaesthesia through a small corneal stab incision, washing out the cortical remnants from behind the posterior capsule. The posterior capsule can be polished using a sandblasted cannula.

\section{Results}

There were 140 eyes with a follow-up from 1 week to 17 months. The average time since surgery was 6.5 months. The average age of the patient was 69 years, with a range from 2 to 93 years.

The designs of lens used comprised 18 Binkhorst posterior chamber lenses (Binkhorst 4-loop lens with the posterior loops excised), 18 Pearce bipod lenses, and 104 Pearce tripod lenses. Two patients had combined keratoplasty and posterior chamber lens insertion of the bipod design, and an additional patient with prior penetrating keratoplasty for conical cornea. Sixty-nine eyes had 2 peripheral iridectomies, and in 71 eyes the peripheral iridectomy was omitted.

\section{VISUAL RESULTS}

One hundred and twelve eyes have an average unaided visual acuity of $6 / 12 ; 103$ eyes have an average visual acuity of $6 / 9$ or better with their present up-to-date refraction, and these eyes also have a best recorded visual acuity of 6/9 from hospital records; $73 \%$ of cases see $6 / 9$ or better with or without spectacle correction.

Refractive state. The average spherical lens requirement taken from the refraction has been $\pm 1 \cdot 1$ dioptres, and of those eyes requiring a cylindrical correction the average astigmatic correction is \pm 1.75 dioptres. This does not include the most recent cases, where the refractive state is changing. In my experience refraction is moderately stable after cataract surgery for 6 weeks.

It is proposed to consider each case with a visual acuity of $6 / 18$ or less individually.

Case 1. The patient had combined penetrating keratoplasty with insertion of a bipod lens for bullous keratopathy. The corrected vision is $6 / 24$, but there are 8 dioptres of corneal astigmatism (the continuous Perlon stitch is still in situ).

Case 2. This patient had been noted to have a prior retinal vascular accident; corrected vision is $6 / 36$ and optic atrophy is present.

Case 3. This patient, with high myopia and myopic fundal changes, has a corrected vision of $6 / 18$.

Case 4. A patient of 82 , who has suffered vitrous loss at operation, and has senile macular degeneration with a corrected vision of 6/18.

Case 5. A patient with corrected vision of $6 / 18$; there is no obvious reason for the poor visual acuity in this case.

Case 6. A patient who had bullous keratopathy, secondary cataract, and a soft eye after an Elliott's trephine operation many years ago. Surgery included penetrating keratoplasty, cataract surgery reconstruction of pupil, and insertion of bipod lens. The corrected vision is $6 / 60$ with a clear corneal graft.

Case 7. This patient had a corrected vision of $6 / 18$, but the intraocular lens is probably centred too high.

\section{Discussion}

Operative complications have been minimal in this series, the greatest problem being removal of all 
cortical material, especially with an immature cataract. There seems to be no way to reduce this time-consuming procedure without expensive aspiration/irrigation equipment. Maintenance of corneal clarity by continuous irrigation with balanced salt solution and availability of balanced salt solution incorporating glutathione and adenosine may in future further reduce postoperative striate keratitis.

The operative techniques described, incorporating dislocating the nucleus into the anterior chamber and its removal with a vectis, can be carried out with a small 9-mm incision. The lens can, indeed, be inserted through a smaller incision than this in the case of secondary insertion or if phacoemulsification equipment is available. Strong mydriatics can be used pre- and postoperatively as the iris sphincter is not involved in supporting the intraocular lens and their use also facilitates absorption of any cortical remants. Return of visual function is rapid, and the increased safety of a small corneoscleral incision allows early hospital discharge (normally the day after operation).

Surgical uveitis has been minimal, and the total absence of postoperative hyphaema is probably related to the abandonment of peripheral iridectomies. The lens design with the 4-mm optic seems to reduce the likelihood of pupil-block glaucoma, there being no such problem in this series, the thin lenticulus allowing adequate drainage between the pupil sphincter margin and the anterior surface of the prosthesis. Increasing the optic size may increase the possibility of pupil-block glaucoma as well as increasing the weight of the lens.

The series has been free of retinal detachment, but there have been two cases with corneal changes, probably due to surgical trauma. The one case of clinical cystoid macular oedema occurred where vitreous loss with rupture of the posterior capsule was a surgical problem. In my own series of 360 eyes with pupillary lenses cystoid macular oedema occurred in 7 eyes $(1.9 \%)$. Binkhorst et al. (1976) maintain that posterior capsulotomy seems to compromise the protection provided by the capsule to the macula.

Cystoid macular oedema would seem now to be the most feared common complaint of implant surgery. Meredith et al. (1976), considering a series of classical intraocular cataract extractions, found cystoid macular oedema occurring in $10 \%$ of eyes. These eyes had a visual acuity of less than $20 / 40$.

Dallas (1976) found that in 260 eyes having had plain cataract extraction cystoid macular oedema occurred in $5(1.8 \%)$. Of 200 eyes with iris clip lenses $12(6 \%)$ suffered macular disturbance. Marmion (1976) emphasised the important prog- nostic implication of the Irvine-Gass type of cystoid macular oedema occurring as a transient episode in about $40 \%$ of cataract extractions, and having a relatively good prognosis. He reviewed 750 cases of classical cataract extraction, of which $2.1 \%$ exhibited this syndrome. In pseudophakia, however, there is a greater preponderance of Jaffe's syndrome, in which there are definite cystoid changes without oedema and with a less favourable prognosis. Both Dallas (1976) and Binkhorst et al. (1976) consider that extracapsular techniques confer some protection to the macular region.

The initial results of this series compare favourably with those of other methods of cataract surgery with or without implantation. Dallas (1976) found that $67 \%$ of eyes after plain cataract extraction saw 6/12 or better. In my review (Pearce, 1975) of Choyce's cases of Mark VIII implants $73 \%$ saw 6/9 or better, and similarly in reviewing Binkhorst's cases of 4-loop pupillary lenses with intracapsular extraction (Pearce, 1972) $67 \%$ of eyes had visual acuity of $6 / 9$ or better.

It is important not to give a prescription for glasses until 6 weeks after surgery. Singh et al. (1976) in their series of plain cataract surgery showed that 3 sutures gave the least astigmatism and that refraction was not stablised for 6 weeks after surgery. The average cyclinder on retinoscopy was 1.77 dioptres. They also found that keratometry bore little relation to the refraction result in their cases. On reviewing my series of 360 eyes with pupillary lenses I found that the spherical correction was \pm 1 dioptre, the cylindrical correction being 1.0 dioptre. It will be interesting in the future to evaluate whether reduction of incision size to $9 \mathrm{~mm}$ with 3 preplaced corneoscleral sutures reduces the astigmatic error after surgery.

Various configurations of lens design are now available (Fig. 1 $a, b, c$ ) and provided there is an adequate inferior capsular bag the $4 \cdot 7-\mathrm{mm}$ radial tripod lens can be used without an iris suture fixation. Nevertheless, iris fixation gives some stability to the lens and may indeed 'polish the posterior capsule' during normal pupillary function.

Patient satisfaction with the operation has been good, and pupillary function is normal, without complaints of photophobia. (These were especially noted when patients have had a posterior chamber lens with a normally acting pupil in one eye and in the other eye an iris clip lens.) The eye itself looks normal (Fig. 2), and the pupil can be fully dilated for examination of the periphery of the fundus (Fig. 3). It would also seem that there is less tendency for pigment deposit on the anterior surface of the prosthesis than with pupillary lenses, and, of course, there is no danger of erosion of the iris sphincter by 


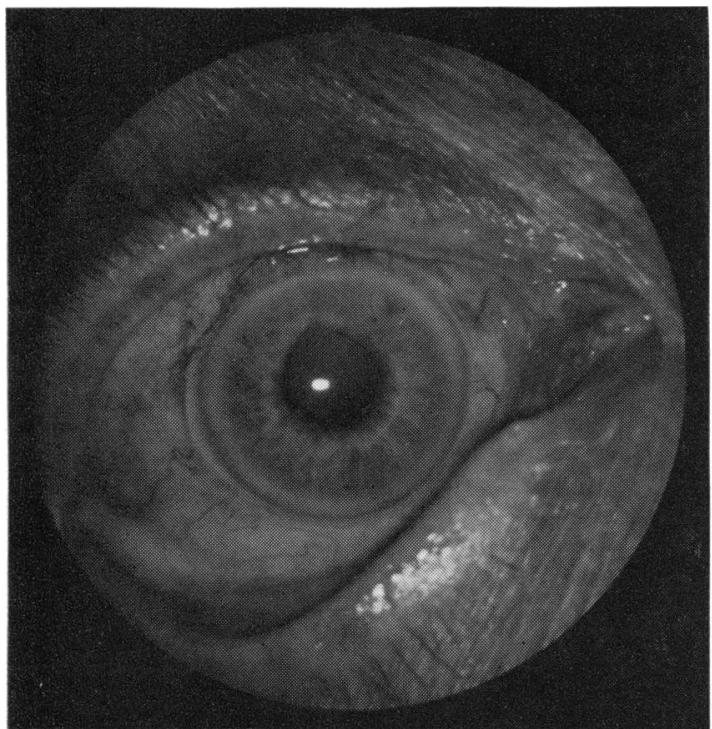

Fig. 2

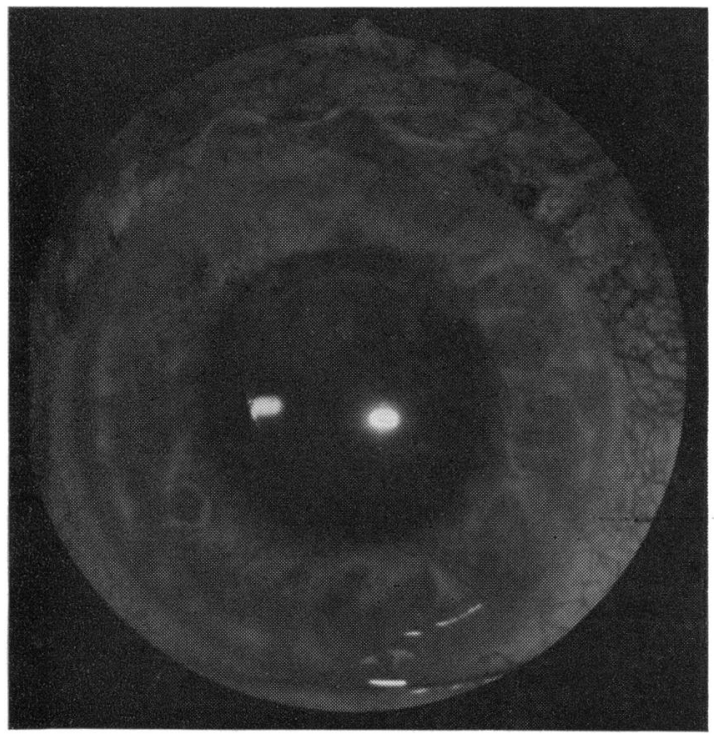

Fig. 3

the posterior loops of an iris clip or iridocapsular lens.

Secondary insertion through a very small incision of about $5 \mathrm{~mm}$ can be easily performed when there is an intact posterior capsule or adequate posterior capsule for prosthesis fixation. In these cases, especially with the younger patient, it is politic to select the bipod lens, which is also the lens of choice for combined keratoplasty. It is wise to have available lenses with varying lengths of inferior feet, so that if the pupil is small after extracapsular extraction a lens with $4 \mathrm{~mm}$ radial inferior limbs can be used. This allows minimal manipulation within the anterior chamber. Conversely, if the pupil remains large, then a longer length of inferior feet is chosen. The pupil should be round at the end of the operation; if it is still widely dilated acetylcholine drops should be instilled before the eye pad is placed. Dilatation of the pupil can then be begun at the first dressing.

Complications of Ridley's original posterior chamber lens, which weighed $106 \mathrm{mg}$ in air and was $8.35 \mathrm{~mm}$ in diameter (approximately $1 \mathrm{~mm}$ smaller than the normal crystalline lens), have been summarised by Ridley $(1953,1954,1958,1960)$ as: iritis; occlusion of the pupil by dense inflammatory membrane; late thickening of the posterior capsule, especially in young patients; anterior chamber loss for unknown reasons; secondary glaucoma due to shallow anterior chamber; iris atrophy; and posterior dislocation of the prosthesis. These complications were not evident in the present series of posterior chamber lenses, so it would seem fair to conclude that the use of a lightweight posterior chamber lens with extracapsular cataract extraction is a relatively safe procedure.

I thank Mr E. Ford, Raynor \& Keeler Ltd, for his continuing help in the development of lenses and for his line drawings, and Miss J. Walden for her secretarial help.

\section{References}

Binkhorst, C. D., Kats, A., and Tijan, L. M. (1976). Transactions of the American Academy of Ophthalmology and Otolaryngology, 81, 123-125.

Dallas, N. L. (1976). Proceedings of the Royal Society of Medicine, 69, 904.

Marmion, V. J. (1976). Proceedings of the Royal Society of Medicine, 69, 904

Meredith, T. A., Kenyon, K. R., Singerman, L. J., and Fine, S. L. (1976). British Journal of Ophthalmology, 60 , 765-769.

Pearce, J. L. (1976). Transactions of the Ophthalmological Societies of the United Kingdom, 96, 6.

Pearce, J. L. (1975). British Journal of Ophthalmology, 59, 99.

Pearce, J. L. (1972). British Journal of Ophthalmology, 56, 328.

Ridley, H. (1953). Transactions of the American Academy of Ophthalmology and Otolaryngology, 57, 98-106.

Ridley, H. (1954). British Journal of Ophthalmology, 38, 156-162.

Ridley, H. (1958). Transactions of the Ophthalmological Societies of the United Kingdom, 78, 585-592.

Ridley, H. (1960). British Journal of Ophthalmology, 44, 705712.

Singh, D., and Kumar, K. (1976). British Journal of Ophthalmology, 60, 640 . 